\title{
IMPROVED CHANNEL ESTIMATION IN TRANSMIT DIVERSITY MIMO-OFDM USING PARTICLE SWARM OPTIMIZATION
}

\author{
Parminder Kaur ${ }^{1}$, Karamjit Kaur ${ }^{2}$ \\ ${ }^{I}$ Student, Electronics and Communication Department, Punjabi University, Patiala, Punjab, India \\ ${ }^{2}$ Assistant Professor, Electronics and Communication Department, Punjabi University, Patiala, Punjab, India
}

\begin{abstract}
This paper proposes a new method of using particle swarm optimization for channel estimation in transmit diversity. Various channel estimation techniques have been studied. The Least Square estimator performance is compared with Adaptive Regularized Least Square estimator receiver which uses output signal as feedback to enhance the Mean Square Error of system to make it closer to ideal performance value. In channel, it is required to transmit the data from transmitter to receiver but sometimes noise is added to the data that has to be transmitted and that noise is called the interference. To improve the estimation process, evolutionary algorithm like optimization techniques can be utilized for the estimation of the channel. Therefore, a method can be developed based on Particle Swarm Optimization based Least Square estimator for transmit diversity case in OFDM. The results have been shown in support of the proposed method.
\end{abstract}

Keywords-MIMO, OFDM, LS, PSO

\section{INTRODUCTION}

Orthogonal Frequency Division Multiplexing (OFDM) is a digital multi-carrier modulation method and is becoming a very popular modulation scheme for transmitting the signals over the wireless channels. This method is being extensively used in wireless communication field because of its higher spectrum efficiency, faster transmission speed and resistance to delay due to multipath components. It has broad applications likewireless LAN, digital television (DMB, DVB), LTE, ADSL etc.[1]

In this new information age, many applications demand the high data-rate wireless access. Conventionally, more bandwidth is needed for high data-rate transmission. However, the increased bandwidth is sometimes expensive or usually impractical. In this case, multiple transmit and receive antennas can be used for bandwidth efficient transmission. The combination of OFDM and Multiple Input Multiple Output (MIMO) systemsprovide expanded capacity and improved quality of service[2]. In this, the whole channel is divided into many sub-channels and signals are transmitted in parallel, thus attaining high data-rate and increased symbol duration to combat Inter-symbol Interference (ISI). Space time block coded (STBC) are used to increase the diversity gain by sending the several copies of the data over the number of antennas and then the reliability of the link is improved by exploiting the various received versions of data. The major challenge faced in MIMO-OFDM wireless system is channel estimation. In wireless systems, channels are frequency-selective and time varying in nature. The transmitted signals goes through the various effects such as reflection, diffraction, multi-path fading etc. The effects of the channel on its response have to be known and it is known as channel state information. In any communication system, channel is to be estimated. This estimation in channel is required for decoding and equalization of the message, thus it can be said that channel estimation is the most significant part in any communication system. The information about channel can be acquired through various methods like training based, blind and semiblind channel estimation methods [3]. Blind channel estimation techniques use the analytical information of the channel and various characterstics of the sent signals. There is no additional loss in this method and is applicable to slow time-variant channels as it needs long data records. Channel estimation using training based method involves pilot symbols or known symbols to be inserted into the symbol stream. Semi-blind channel estimation method is a combinational technique because it employs both the training and blind techniques.

Training-based channel estimation uses two types of pilot arrangements that is, Comb-type and Block-type. In block type, the pilots are introduced into all sub-carriers whereas in case of comb-type, the pilots are introduced only in certain sub-carriers. In this paper, comb-type pilot arrangement is employed. There are various channel estimation algorithms like Least Square (LS), Minimum Mean Square Error (MMSE) Estimator, Regularized Least square (RLS),[4] [5] etc. In this paper, LS and RLS are studied and a new channel estimation methodusing Particle Swarm Optimization (PSO) has been advocated to find optimum solution for channel estimation. The performance of three estimators is then compared with parameters like Mean Square Error (MSE) and Signal-to-Noise Ratio (SNR). Further the next sections are organized as follows: The section 2 depicts the MIMOOFDM system model. The LS and RLS are briefly explained in section 3. Section 4 discusses the proposed algorithm for MIMO-OFDM systems. Section 5 shows the simulation results and discussions. Section 5 finally ends the research paper. 


\section{SYSTEM DESCRIPTION}

A model with ' $m$ ' transmitter antennas and one receiving antenna is given in Fig 1. At time step n, a data block $\{b(n, k), k=0,1, \ldots . . N-1\}$ where $\mathrm{N}$ is the number of subcarrier, can be divided into $\mathrm{m}$ different symbol blocks
$\left\{X_{i}(n, k), k=0,1, \ldots . . N-1, i=1, \ldots \ldots . m\right\}$. Each symbol block is sent on $\mathrm{N}$ sub-carriers over different antennas. Communication link is build-up between every pair of transmitter and receiver antennas.
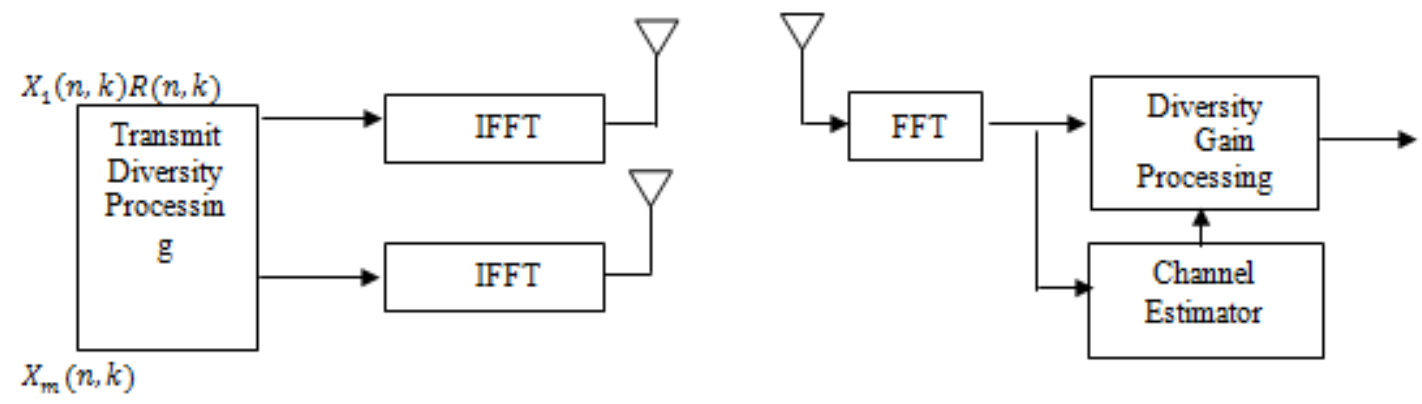

Fig.1 MIMO-OFDM system model

The ' $\mathrm{m}$ ' distorted signals superimposed on each other on $\mathrm{N}$ sub-carriers after demodulation and are received on receiver side. The coefficient for impulse response of channel from the ith transmitting is given by [6]:

$$
\begin{gathered}
h_{i}(n, \tau)=\sum_{l=0}^{L-1} h_{i}(l) \exp \left(j \frac{2 \pi}{N_{s}} f_{D l, i} T_{n}\right) \delta\left(\tau-\tau_{i, l}\right) \quad i \\
=1, \ldots \ldots, m
\end{gathered}
$$

Here, $\tau$ is the delay range, $\tau_{i, l}$ represents $/$ th-path delay time, $f_{D l, i}$ is the $l$ th-path Doppler frequency shift which generates ICI in the received signals. $h_{i}(l)$ is the complex frequency response of the $l$ th path. When the messages from ' $m$ ' transmitter antennasare sent over the channel with additive Gaussian noise then the received OFDM signal in time domain is given by:

$$
y(n)=\sum_{i=1}^{m}\left(x_{i}(n) \otimes h_{i}(n, \tau)\right)+w(n)
$$

Here, $n=0,1, \ldots \ldots . . .1$ and $w(n)$ is the noise. FFT transform of the signal is done at receiver side on the $k$ th sub-carrier and given by:

$$
Y(n, k)=\sum_{i=1}^{m}\left(X_{i}(k) H_{i}(k)+I_{i}(k)+W_{i}(k)\right.
$$

Here, $k=0,1, \ldots \ldots . N-1$ and $H_{i}(k)$ represents coefficient ofchannel impulse responsefrom the ith transmitter antenna to the receiver antenna on the $k$ th sub-carrier, during the symbol duration of $n$th OFDM.

\section{CHANNEL ESTIMATORS}

\subsection{Least Square (LS) Estimator}

This method estimates the parameters by minimizing the squared discrepancies between the observed values and the expected values. The LS estimator works for above mentioned minimization as:

$$
(Y-X H)^{H}(Y-X H)
$$

Where $(\ldots)^{H}$ is a hermitian matrix ,Y represents received data, $\mathrm{X}$ is the transmitted data and $\mathrm{H}$ is the channel response. The LS estimation of $\mathrm{H}$ can be expressed as:

$$
\hat{H}_{L S}=X^{-1} H
$$

\subsection{Regularized Least Square (RLS)}

Adaptive channel estimation algorithms are gaining more attention these days. As the wireless channel is time varying and has random nature. Therefore adaptive algorithms are best in order to keep track of it. The adaptive algorithms continuously update their parameters until they attain the optimum solution. In RLS the past or previously estimates are used to calculate the new estimates.[5]

\section{PSO BASED PROPOSED ALGORITHM}

Particle swarm optimization (PSO) is advanced, metaheuristic, evolutionary optimization algorithm, founded in 1995 by Dr. Eberhart and Dr. Kennedy, invented by observing the collaborative behavior of bird flocks or school of fish to direct particles to search for global optimum solutions.[7] PSO has many likenesses with generative computational algorithms such as Genetic Algorithms (GA). The system starts with inputting thenumber of random particles and explore for optimum solution by revising the values ofparticles. However, PSO has no transformation operators which are needed in GA. So the main benefits of PSO is ease in implementation and adjustment of limited parameters.

In PSO,once the particles are initialized then these particles travel the problem space by following the path of current optimum particles. Each particle maintains record of its position in the search space of function which describes the 
best solution (fitness) attained so far. Thisbest solution value is also recorded.Each particle searches for better position in problem space by changing velocity and position. Initially, velocity and position are given the random values in problem space. The position and velocity of particle ion dimension $\mathrm{d}$ are then updated on each iteration as shown below:

$$
\begin{gathered}
v_{i}(t+d t)=w * v_{i}(t)+c_{1} * r_{1} *\left(\text { pbest }_{i}(t)-x_{i}(t)\right)+c_{2} \\
* r_{2}\left(g b e s t(t)-x_{i}(t)\right) \\
x_{i}(t+d t)=x_{i}(t)+v_{i}(t) d t
\end{gathered}
$$

Here, pbest $t_{i}$ represent the best position acquired by ith particle and gbestrepresent the best position among all particles. $c_{1}, c_{2}$ are cognitive and social parameters whereas $r_{1}, r_{2}$ are random vectors with values between ' 0 ' and ' 1 ' and $\mathrm{w}$ isinertia weights which is used to maintain momentum of particle[8]. The inertia weight aids in controlling the effect of previous velocity values on the current velocity, therefore gives the trade-off between the global and local searches. A large inertia weight makes easy a global search and a small inertia weight gives the facility of local search. Thus, that value of the inertia weight is to be selected which provides a balance between global and local searches. By this optimum solution is obtained in less number of iterations on the average.For optimalperformance, acceleration constants $c_{1}$ and $c_{2}$ should be kept small, which however affects the convergence rate. So, the choice of acceleration constants varies from application to application. This optimization algorithm finally converge when each particle reaches the global best or the present extreme value of the cost function.

Steps: The various steps for proposed algorithm are given below:

1. MIMO-OFDM channel estimation is started by selecting 2 transmitters and 1 receiver.

2. QPSK modulation scheme is adopted with 64 subcarriers for transmission purpose.

3. Additive White Gaussian Noise is added to the output and channel is estimated using Least Square channel estimation.

4. Using the same parameters, channel is estimated using Adaptively Regularized Least Square method.

5. Particle Swarm Optimization technique is applied to the Least Square channel estimation method to find the optimum solution.

The flow chart for PSO algorithm is given below:

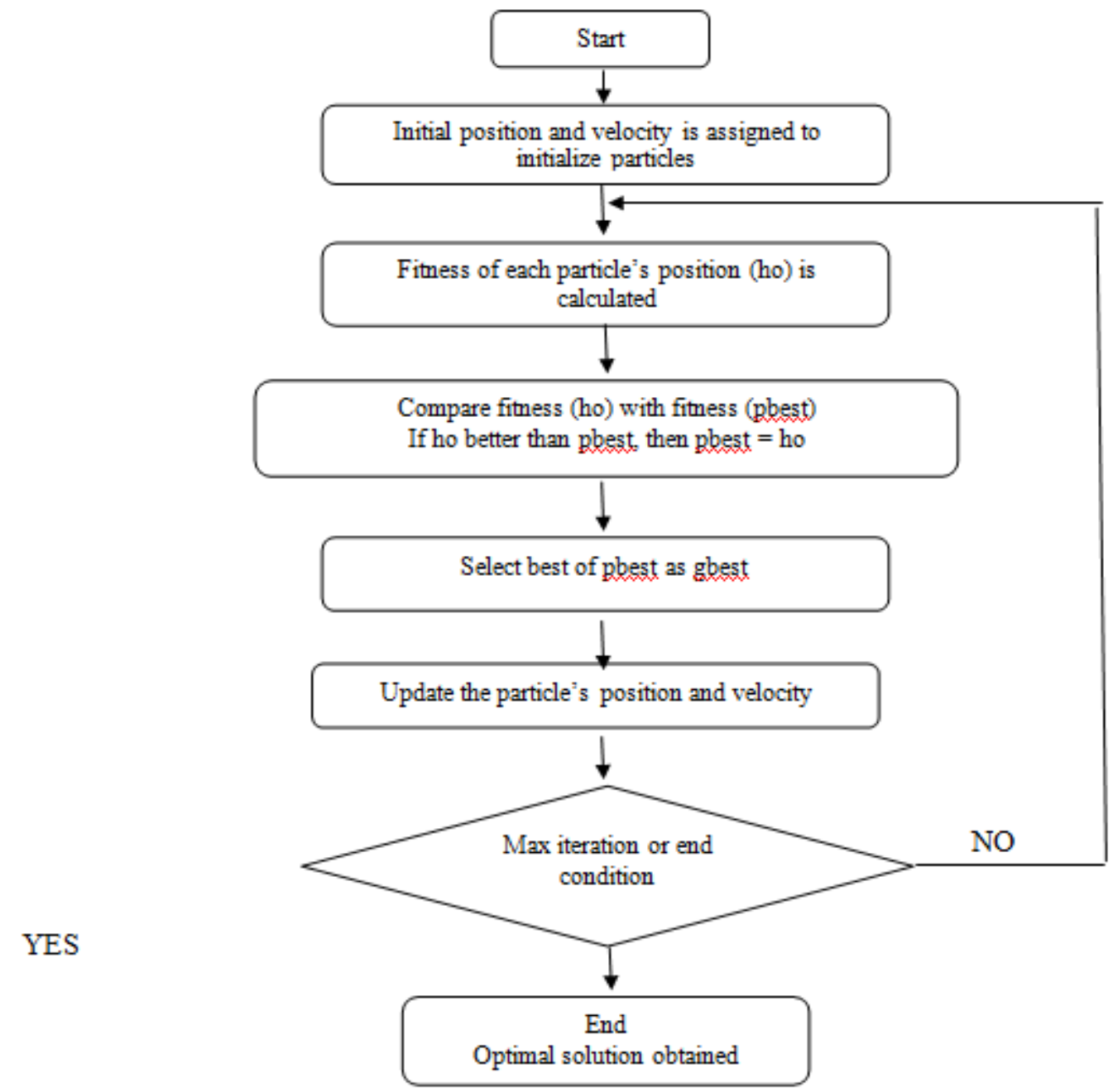

Fig 2. PSO flow chart 


\subsection{Performance Parameters}

1. Mean Square Error: It is the average of the squares of the errors or deviations, that is, the difference between the observed and expected value. The MSE of an estimator $\hat{h}$ with respect to an unknown parameter $h$ is defined as:

$$
\operatorname{MSE}(\hat{\mathrm{h}})=\mathrm{E}\left[(\hat{\mathrm{h}}-\mathrm{h})^{2}\right]
$$

2. Signal to Noise Ratio: Signal to noise ratio is represented as the ratio of total signal power to the total noise power, usually in decibels. It is a measure of useful information with respect to irrelevant information.

\section{RESULTS AND DISCUSSIONS}

To investigate the channel estimation error in MIMOOFDM system, computer simulations are performed using MATLAB. The number of sub-carriers and FFT points are taken to be 64. Rayleigh, Rician and Flat fading channels are constructed for simulations. Simulations are carried out for QPSK technique. Performance is compared for LS, RLS and PSO techniques. The following graphs show the graphs for channel MSE versus SNR.

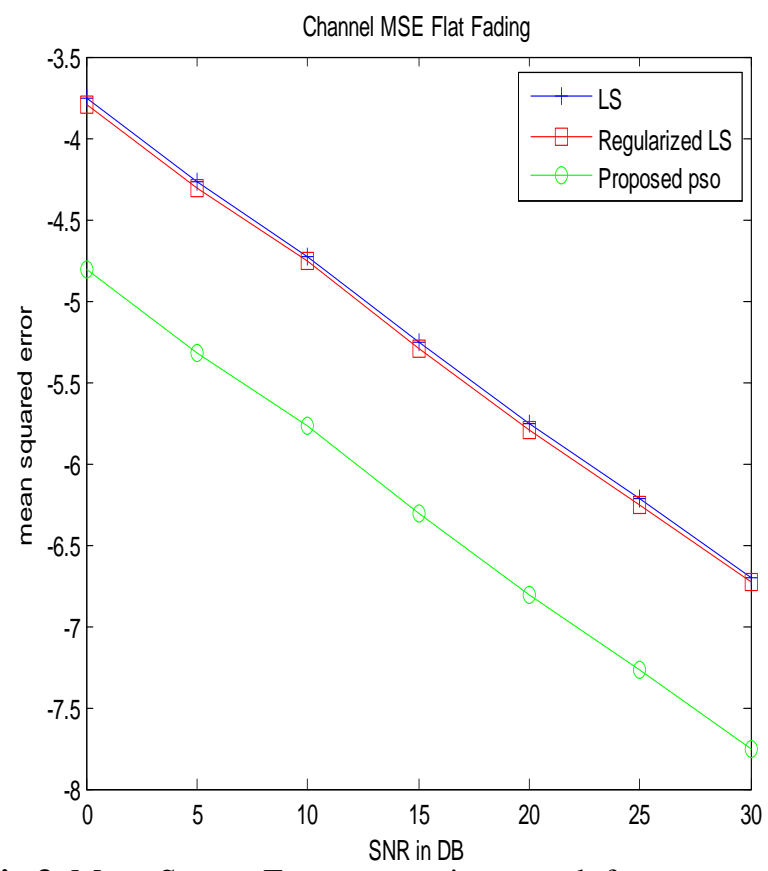

Fig 3. Mean Square Error comparison graph for proposed as well as existing techniques forflat fading

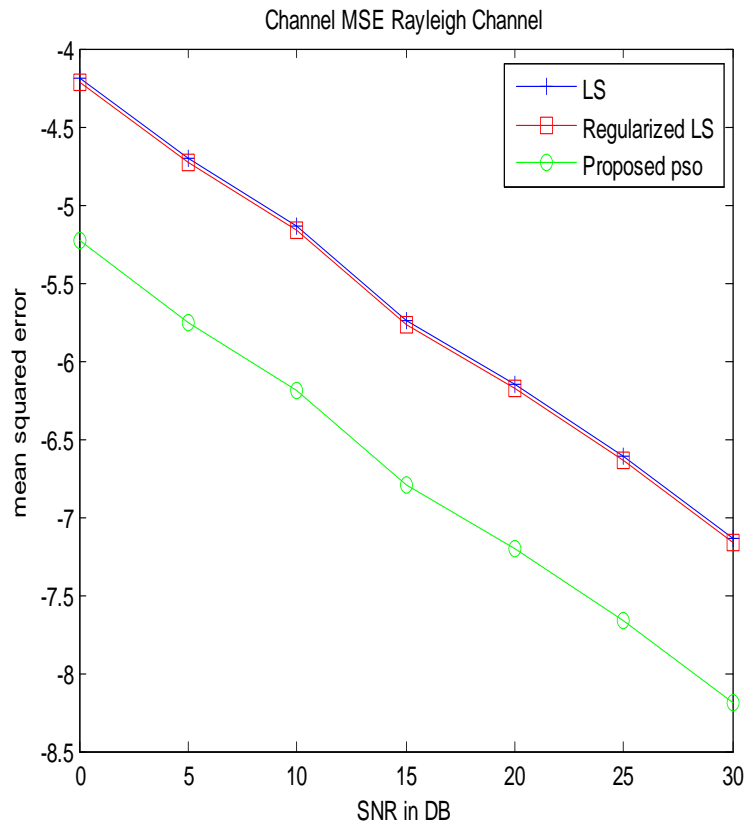

Fig 4. Mean Square Error comparison graph for proposed as well as existing techniques over Rayleigh fading channel

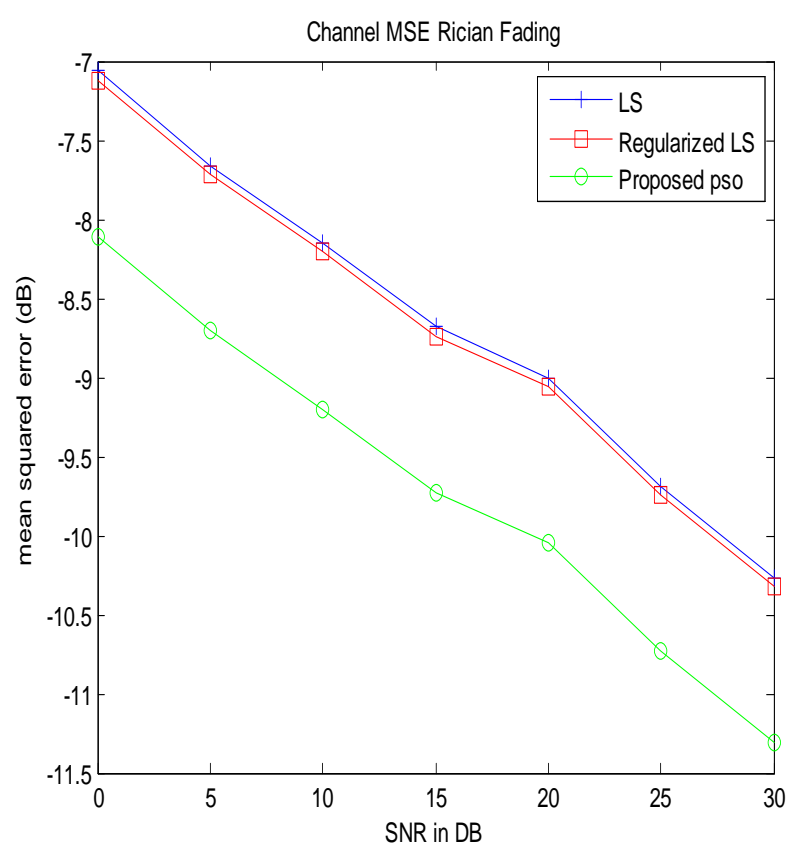

Fig 5. Mean Square Error comparison graph for proposed as well as existing techniques for Rician fading channel

\section{CONCLUSION}

In this work, the various channel estimation techniques for MIMO-OFDM are studied and in order to optimize the estimation in Transmit Diversity case, a new Channel estimation method using Particle Swarm Optimization (PSO) has been advocated. Performances of Channel estimation algorithms have been compared and it can be seen from the results that least square algorithm is the simplest amongst all but has low performance. The adaptively regularized method provides slight better performance over the existing LS technique in flat fading 
and implements a slightly complex method. Also, the regularized method has low performance in Rayleigh and Rician channels. The complexity of proposed system is almost similar to that of the adaptively regularized method but has performed better than both the methods. Hence the proposed method can be considered for practical implementation. Further, a MSE comparison of the method is also shown for all the three methods and it can be concluded that the proposed method has performed slightly better in comparison to other methods.

\section{REFERENCES}

[1] IEEE Computer Society LAN MAN Standards Committee. "IEEE 802.11: Wireless LAN medium access control and physical layer specifications," 1999.

[2] Ganesh, R.S., J. Jayakumari, and L.P. Akhila., "Channel estimation analysis in MIMO-OFDM wireless systems," IEEE International conference on Signal Processing, Communication, Computing and Networking Technologies, pp. 399-403, 2011.

[3] O.Edfors, M. Sandell, J. J. Van de Beek, S. K. Wilson and P. O. Boriesson, "Analysis of DFT-based channel estimators for OFDM." Vehicular Technology Conference, July 1995.

[4] S. Saleem and Qamar-ul-Islam, "Optimization of LSE and LMMSE channel estimation based on CIR samples and channel taps," International Journal of computer Science Issues, Vol. 8, Issue 1, January 2011.

[5] K. Wu and M. chang, "Adaptively regularized least square estimator for decision-directed channel estimation in transmit-diversity OFDM systems," IEEE wireless communications letters, vol.3, NO. 3, June 2014.

[6] Y. Qiao, S. Yu, P. Su and L. Zhang, "Research on iterative algorithm of LS channel estimation in MIMOOFDM systems," IEEE Transactions on Broadcastings, vol. 51, NO. 1, March 2005.

[7] J. Kennedy and R. Eberhart, "Particle swarm optimization," in Proceedings of the IEEE International Conference on Neural Networks, pp. 1942-1948, Perth, Australia, December 1995.

[8] YH Shi and RC Eberhart, "A modified particle swarm optimizer," Proceedings of 1998 IEEE International Conference on Evolutionary Computation, pp. 69-73, 1998. 